LBNL-51583

\title{
MEASURING RATES OF OUTDOOR AIRFLOW INTO HVAC SYSTEMS
}

\author{
William J. Fisk, David Faulkner, Douglas P. Sullivan, and Woody Delp \\ Indoor Environment Department \\ Environmental Energy Technologies Division \\ Lawrence Berkeley National Laboratory \\ Berkeley, CA 94720
}

\section{Acknowledgements}

This work was supported by the assistant Secretary for Energy Efficiency and Renewable Energy, Building Technologies Program of the U.S. Department of Energy under contract DE-AC03-76SF00098. The authors thank Mike Apte and Craig Wray for their reviews of a draft of this document. 


\title{
MEASURING RATES OF OUTDOOR AIRFLOW INTO HVAC SYSTEMS
}

\author{
William J. Fisk, David Faulkner, Douglas P. Sullivan, and Woody Delp \\ Indoor Environment Department \\ Environmental Energy Technologies Division \\ Lawrence Berkeley National Laboratory \\ Berkeley, CA 94720
}

\begin{abstract}
During the last few years, new technologies have been introduced for measuring the flow rates of outside air into HVAC systems. This document describes one particular technology for measuring these airflows, a system and a related protocol developed to evaluate this and similar measurement technologies under conditions without wind, and the results of our evaluations. We conclude that the measurement technology evaluated can provide a reasonably accurate measurement of OA flow rate over a broad range of flow, without significantly increasing airflow resistance.
\end{abstract}

\section{Background}

Ventilation, i.e., providing outdoor air (OA), has a substantial influence on building energy consumption, occupant health, and occupant satisfaction with the indoor environment. The quantity of energy used for ventilation in the U.S. service sector (i.e., commercial, institutional, and government buildings) is uncertain, but clearly substantial. Orme (1998) estimated that the energy consumed to ventilate these buildings is 1.4 Quad (1.5 EJ), which is approximately one quarter of the total service-sector building energy use; however, this estimate is likely to be too high because it assumes continuous occupancy. Through more detailed analyses with estimates of actual periods of occupancy, Emmerich and Persily (1998) predicted that 0.13 Quad (0.14 EJ) of heating and cooling energy would be used for ventilating U.S. office buildings, if all offices had a ventilation rate of $20 \mathrm{cfm}\left(10 \mathrm{~L} \mathrm{~s}^{-1}\right)$ per person. Applying the ventilation energy use per unit floor area of Emmerich and Persily (1998) to the entire stock of U.S. service sector buildings yields an estimated energy consumption for ventilation of 0.71 Quad $(0.75 \mathrm{EJ})$ for the entire stock. We expect that the actual energy use is considerably higher, i.e., between 0.71 and 1.4 Quad ( 0.75 and $1.5 \mathrm{EJ})$, because many types of service sector buildings have a higher occupant density or are ventilated for longer periods of each day than offices.

The scientific literature describing the influence of ventilation rates on health and occupant satisfaction was reviewed by Seppanen et al. (1999). On average, lower ventilation rates were associated with increased prevalences of communicable respiratory illnesses (e.g., common colds), increased prevalences of sick building syndrome (SBS) symptoms, and diminished satisfaction with indoor air quality. A large majority of studies reviewed found statistically significant and substantial $(10 \%$ to $>100 \%)$ increases in health outcome prevalences with lower ventilation rates. The evidence of adverse effects was strongest when ventilation rates were reduced below $20 \mathrm{cfm}\left(10 \mathrm{~L} \mathrm{~s}^{-1}\right)$ per person, which is the current minimum rate for offices in 
ASHRAE Standard 62 (ASHRAE 2001); however, several studies reported benefits of increasing ventilation rates above $20 \mathrm{cfm}\left(10 \mathrm{~L} \mathrm{~s}^{-1}\right)$ per person.

Despite the substantial influences of ventilation rates on energy use and health, very few U.S. buildings have an integral system for measuring ventilation rates. The typical practice in service sector buildings, which are the primary focus of this report, is to have an air balance company measure the OA flow during a period of building commissioning or airflow balancing and adjust the positions of the dampers for OA, recirculation air, and exhaust air to obtain the desired minimum rate of OA supply. However, accurately measuring OA airflow into HVAC systems is technically challenging and typical practices do not always satisfactorily determine minimum ventilation rates. For example, the minimum OA flow is sometimes based on the difference between supply and return air flow rates. Even with perfect measurements, the estimate will often not be valid because there is significant airflow through the exhaust damper system (Krarti et al. 1999). Also, modest errors in measurements of supply and return air flow rates can produce a large error in the difference between these two flow rates (Kettler 1995).

An alternative method for measuring OA flows is a velocity traverse with a rotating vane anemometer at the exterior face of the outdoor air inlet. However, the non-stable (due to winds) and spatially non-uniform air velocities at the outdoor air intakes, plus the difficulty in determining the effective area of the intake can result in large errors (Howell et al. 1987, Krarti et al. 1999).

Another alternative measurement approach is to determine the outdoor airflow from the product of a measured supply airflow rate and the percentage of outdoor air $(\% \mathrm{OA})$ in the supply air stream, which is determined from air temperature measurements and an energy balance calculation. However, non-uniform airstream temperatures and small differences between temperatures can cause large errors in the estimated \%OA and associated OA flow rate (Krarti et al. 1999).

Even if air balance professionals could provide perfect measurements of OA flow rates during their occasional visits to buildings, the OA flow is not stable. The actual rates of OA flow will often vary with changes in wind and as the supply air flow rates of variable air volume (VAV) HVAC systems are modulated. In addition, minimum damper positions, which affect OA flow rates may change from those set by the air balance professional due to deliberate adjustments by building operators and to wear or failures in the damper actuators and linkage.

Given these measurement challenges it is not surprising that the ventilation rates measured in surveys by researchers using tracer gas based measurement systems (e.g., Turk et al. 1989, Lagus Applied Technologies 1995, Persily 1989) often differ substantially from the minimum ventilation rates specified in the applicable codes. Many buildings have minimum ventilation rates far exceeding code requirements and a significant portion of buildings provide less ventilation than specified in codes. In some buildings, OA flows and associated building pressures are very poorly controlled. For example, in our field studies it has not been unusual to find indoor air flowing to outdoors through the OA intake louvers and dampers.

There are significant obstacles to cost-effective and accurate measurements of OA flow rates. First, measurements are challenging because OA intake velocities are intentionally kept low in order to prevent rain and snow from being drawn into the air handler. Sizing of the OA air inlet for the entire OA flow into the air handler during economizer operation compounds the problem. The result is particularly low OA intake velocities during periods of minimum OA supply, when measurements are most important. As an example, to prevent entrainment of rain and snow the 
maximum air velocity within the "free area" of an intake louver is usually 400 to $1000 \mathrm{fpm}$ (2 to $5 \mathrm{~m} \mathrm{~s}^{-1}$ ) depending on the design of the intake louver. These velocities occur with the maximum flow at the OA intake during economizer operation with $100 \%$ outdoor air. Since the minimum OA supply is typically about $15 \%$ of the full supply air flow rate, the velocities of OA flowing into the OA intake during periods of minimum OA flow will be only 60 to $150 \mathrm{fpm}(0.3$ to $0.8 \mathrm{~m}$ $\mathrm{s}^{-1}$ ). At these low velocities the dynamic pressure, which is often used in to measure air speed, is only a fraction of a Pascal, which is too low for accurate measurements in field settings.

The geometry of the OA intake and its impact on velocity profiles further complicates the measurements. The outdoor air passes through a bird screen, a set of louvers, and an adjustable outside air damper. Downstream of the louvers or OA dampers the speed and direction of airflow will normally vary markedly across the flow cross section; thus, averaging velocity measurements made at a few locations in the cross section may also lead to large measurement errors. At the exterior face of the OA intake, measurements are very problematic because even normal winds cause a large fluctuation in air velocity. To reduce the obstacles to measurements, components could be added that increase air velocities and straighten the flow downstream of the louvers or OA damper. Also, large numbers of velocity sensors could be used to better assess the average velocity. However, practical measurement systems face cost, pressure drop, and space constraints.

While these problems and the need for better measurement and control of OA ventilation rates have been recognized for many years, until recently there has been little progress toward meeting this need. The review of Krarti et al. (1999) on measurement and control of OA flow in variable air volume systems includes a summary of much of the recent research. In particular, Krarti et al. (1999) point out that the long unobstructed OA ducts needed for most flow rate measurements will generally be impractical, and they identify a few more promising alternatives, including:

1. providing a separate outdoor air duct for the minimum outdoor airflow with air velocities maintained sufficiently high for use of Pitot-static tube arrays;

2. maintaining a constant pressure drop across the OA louvers and dampers during minimum outdoor air conditions; and

3. using a $\mathrm{CO}_{2}$ mass balance to compute the percentage of outdoor airflow (\%OA) and multiplying by the separately metered supply airflow to determine the outdoor airflow.

While each of these alternatives has merit, they also have some drawbacks. Alternative 1 (separate OA ducts) may be unattractive to designers, especially for small to moderate size HVAC systems. Alternative 2, is a flow control strategy but requires a separate measurement system for calibration of flow versus pressure drop. Alternative 3, requires an accurate measurement system for supply flow rates and is not applicable when indoor $\mathrm{CO}_{2}$ concentrations are marginally above outdoor concentrations because of a low occupant density or because occupants arrived recently. Also, alternatives 1 and 2 only provide a measurement during minimum outside air supply.

Within the past few years, manufacturers have pursued another option -- the direct measurement of airflow through the OA intake using a sensor system located at the intake. Such measurements are attractive because no significant change to the HVAC system is required (e.g., no separate OA duct) and because OA flows are measured during both minimum OA supply and during periods of economizer operation with an increased OA supply. A handful of related measurement technologies have emerged on the market within the last few years. 
The objectives of the research discussed in this report are to: 1) evaluate the performance (accuracy and pressure drop) of these emerging direct measurement technologies; and 2) develop a test system and protocol for evaluating measurement system performance. Future objectives will include: 3) helping a professional organization, such as ASHRAE, establish a standard test method that will enable engineers and facility managers to rate and evaluate products; and 4) evaluating some of our own ideas for OA flow measurement technologies. This report focuses on the first two objectives and summarizes our progress to date.

\section{Approach}

\section{Test system description}

The laboratory test system, illustrated diagrammatically in Figure 1, has a changeable OA intake louver and damper system, air recirculation ductwork, a variable speed fan, recirculation and exhaust dampers, and a precision "reference" airflow meter upstream of the location of air exhaust. Technologies for measuring OA flow can be installed per manufacturers specifications. Turning vanes are installed in the bend of the recirculation ductwork upstream of the location where recirculated and outdoor air mix. Independent control of the OA and recirculation air flow rates can be accomplished using the dampers and variable speed fan. Because the system is sealed to prevent air leakage, the flow of OA into the test system equals the exhaust airflow rate, which is measured with the reference airflow meter. Thus, the accuracy of the OA measurement technology being tested is determined by comparison to the reference airflow meter, and the percentage measurement error (\%error) is calculated from the following equation:

$$
\% \text { error }=100 \%\left(Q_{m t}-Q_{r e f}\right) / Q_{r e f}
$$

where $Q_{m t}$ and $Q_{r e f}$ are the OA flow rates from the measurement technology being evaluated and the reference flow meter, respectively.

Static pressure taps are installed at a number of locations to enable measurements of pressure drops across the measurement systems. Per specifications in ANSI/ASHRAE standards (ASHRAE 1999a, ASHRAE 1999b), the taps are 0.07 inch $(1.8 \mathrm{~mm}$ ) diameter holes in the duct wall with a smooth inner face. 


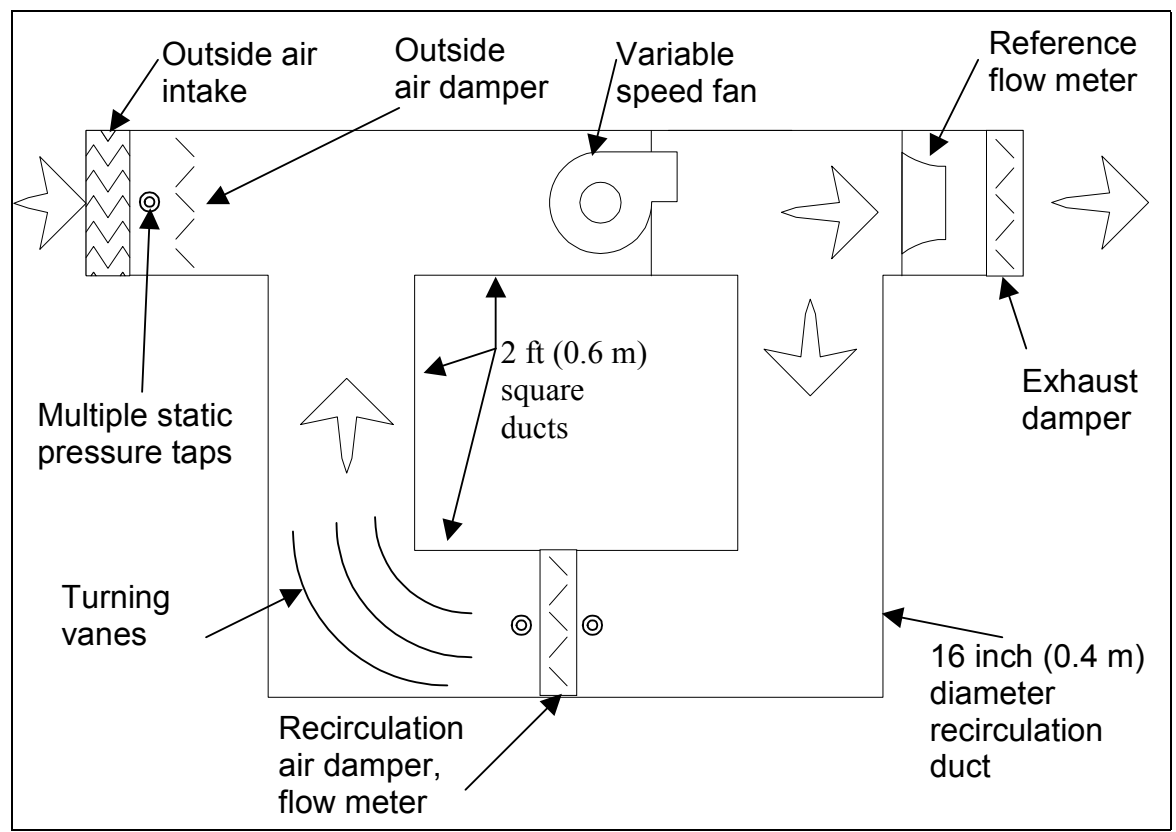

Figure 1. Illustrative diagram of test system

The recirculation flow rate is measured with less accuracy based on the pressure drop across an iris-style damper, relying on the manufacturer's calibration of flow versus pressure drop as a function of damper position.

The output signals of pressure transducers are logged with a personal-computer-based data acquisition system. Instrumentation specifications are provided in Table 1. The calibration of the eight-channel pressure transducer system was checked using a micro-manometer that has a micrometer and electrical circuit for precisely measuring the height of the fluid column. A skilled user of the micro-manometer can obtain measurements repeatable within $0.1 \mathrm{~Pa}$.

The velocity profile of air entering an OA intake may be affected by winds. Our preliminary tests (described subsequently) have indicated that winds can affect the accuracy of OA measurement technologies; however, we are still evaluating methods (that could be incorporated within a practical standard testing method) of producing repeatable simulated winds at the outside air intake. We have also designed our test systems for optional placement on a building rooftop with real winds.

In practice, OA intakes are located near some surfaces such as rooftops, walls of buildings, or walls used as visual (sometimes called architectural) screens, that may affect the entering velocity profiles and the accuracy of OA flow measurement technologies. In the laboratory, we have used movable flat panels to simulate these surfaces. 
Table 1. Instrumentation used with the test system

\begin{tabular}{|c|c|c|c|}
\hline $\begin{array}{l}\text { Parameter } \\
\text { Measured }\end{array}$ & Type of Instrument & Measurement range & $\begin{array}{l}\text { Manufacturer's Rated } \\
\text { Accuracy }\end{array}$ \\
\hline $\begin{array}{l}\text { Exhaust } \\
\text { (reference) } \\
\text { flow rate }\end{array}$ & $\begin{array}{l}\text { Brandt Model NZP1031- } \\
18 \text { "-1-CF and Model } \\
\text { NZP1031-10"-1-CF flow } \\
\text { meters flow meters have a } \\
\text { flow straightener and } \\
\text { converging nozzle, with } \\
\text { Pitot-static type sensor } \\
\text { centered at outlet of } \\
\text { nozzle }\end{array}$ & 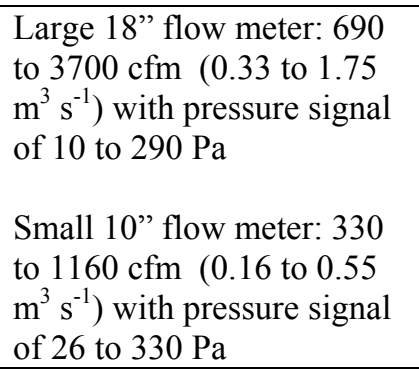 & $0.5 \%$ of reading \\
\hline $\begin{array}{l}\text { Recirculation } \\
\text { flow rate }\end{array}$ & $\begin{array}{l}\text { Aeroflo, Inc } 16 \text { " Iris } \\
\text { Damper with integral } \\
\text { differential pressure flow } \\
\text { meter }\end{array}$ & $\begin{array}{l}150 \text { to } 4000 \mathrm{cfm}(0.07 \text { to } 1.9 \\
\left.\mathrm{m}^{3} \mathrm{~s}^{-1}\right) \text { for differential } \\
\text { pressure range of } 25 \mathrm{~Pa} \text { to } \\
500 \mathrm{~Pa}\end{array}$ & $\pm 7 \%$ of reading \\
\hline $\begin{array}{l}\text { Pressure } \\
\text { difference }\end{array}$ & $\begin{array}{l}\text { Energy Conservatory } \\
\text { Model APT 8, eight } \\
\text { channel electronic } \\
\text { differential pressure } \\
\text { transducer }\end{array}$ & $\pm 400 \mathrm{~Pa}$ & $\begin{array}{l}\text { Larger of } \pm 0.2 \mathrm{~Pa} \text { or } \pm \\
1 \% \text { of reading }\end{array}$ \\
\hline
\end{tabular}

\section{Test protocol}

The protocol for evaluating measurement technologies is still being developed, but is rather straightforward. By adjusting dampers, we varied OA flow rates between approximately 50 and $2,800 \mathrm{cfm}\left(0.02 \mathrm{~m}^{3} \mathrm{~s}^{-1}\right.$ and $\left.1.3 \mathrm{~m}^{3} \mathrm{~s}^{-1}\right)$. Recirculation air flow rates were adjusted so that the percentage of outdoor air $(\% \mathrm{OA})$, [i.e., outdoor air flow rate divided by outdoor plus recirculation flow rate, expressed as a percentage] ranged from $10 \%$ to $100 \%$. To assess the effects of surfaces (walls or a rooftop) near the outdoor air intake, movable flat panels were placed in the three orientations shown within Figure 2, or removed. For some tests, we varied the degree of opening of the OA damper located just downstream of the intake louver. Data were collected with the damper open approximately $100 \%, 50 \%$ and $25 \%$, based on the angle of the shaft that normally connects the damper system to a damper motor.

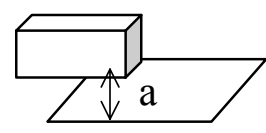

Orientation 1

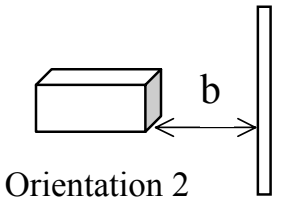

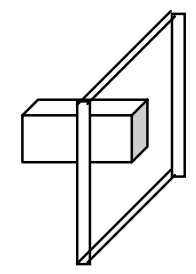

Orientation 3

Figure 2. Orientations of flat panels used to simulate the presence of a rooftop (orientation 1), visual screen (orientation 2) or building wall (orientation 3 ) near the OA intake. $a=1 \mathrm{ft}(0.3 \mathrm{~m})$ and $b \geq 0.33 \mathrm{ft}(0.1 \mathrm{~m})$. The grey shaded surface denotes the OA intake. In orientation 3 , the OA intake was located in the same plane as the flat panel, simulating an OA intake flush with an exterior wall of a building.

Using a fan and flow straightener to simulate steady winds at the OA intake, we performed a very preliminary investigation to determine if winds could affect the accuracy of measurements when there were no simulated surfaces or visual screens near the OA intake. The simulated winds, with a speed range of 2 to $7 \mathrm{mph}$ ( 1 to $3 \mathrm{~m} \mathrm{~s}^{-1}$ ), were oriented directly into the face of the OA inlet and also at 45-degree angles from each side of the OA inlet. 


\section{Measurement technologies}

This report summarizes results of our first evaluation of an OA measurement technology, primarily under conditions without winds at the OA intake. The technology, denoted as MT1 and illustrated in Figure 3 integrates a set of closely spaced vertical louvers with a set of downstream airflow sensing blades that extend over the height of the louver system and that are centered between adjacent blades of the louver. The manufacturer provides a calibration curve in terms of average air velocity through the free-area of the louver system versus pressure signal from the airflow sensing blades. The airflow sensing blades appear to be designed to provide a pressure signal proportional to the average velocity along a vertical path centered between adjacent louvers. Compared to many louver systems, the MT1 louver system also has a relatively high recommended maximum free area velocity which helps to maintain a measurable pressure signal. The shape of the airflow-sensing blade also yields a larger pressure signal than a standard Pitotstatic tube.

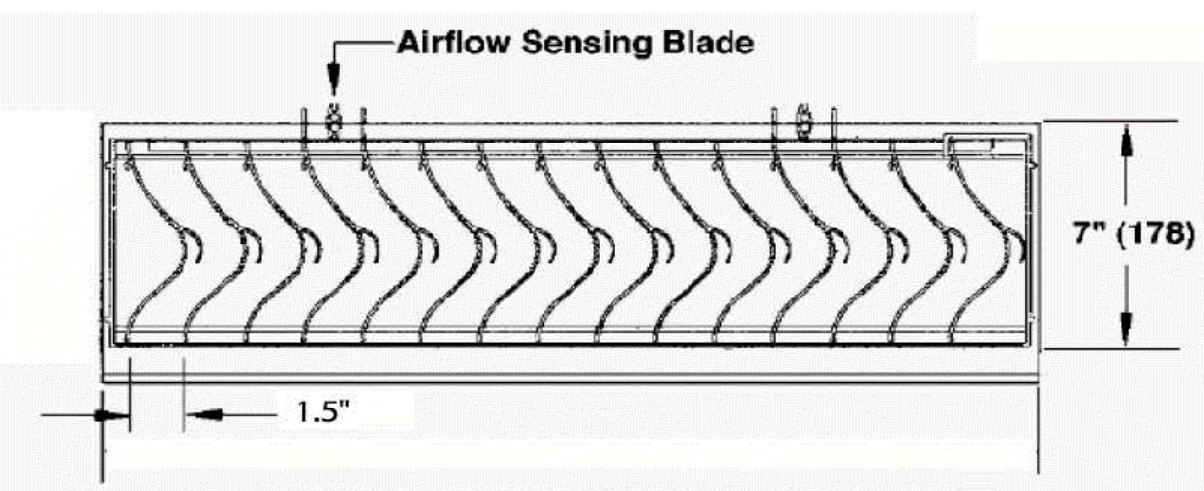

AIRFLOW SENSING BLADE

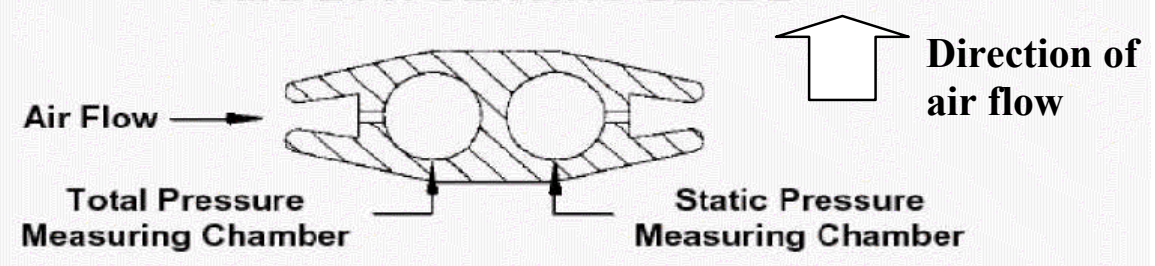

Figure 3. Illustration of outdoor airflow measurement technology number 1 (MT1). Top views of a cross section of the louvers and airflow sensing blades are shown. The airflow sensing blades extend vertically nearly the full height of the louver system.

The tests were conducted with a nominal $2 \mathrm{ft}$ by $2 \mathrm{ft}(0.6 \mathrm{~m}$ by $0.6 \mathrm{~m})$ louver system with two downstream sensing blades. The manufacturer's-reported free area of the $0.6 \mathrm{~m}$ by $0.6 \mathrm{~m}$ louver system is $1.24 \mathrm{ft}^{2}\left(0.12 \mathrm{~m}^{2}\right)$, or $31 \%$ of the nominal face area. The manufacturer's "velocity requirements" for MT1 are 345 to $4000 \mathrm{fpm}\left(1.8\right.$ to $\left.20.3 \mathrm{~m} \mathrm{~s}^{-1}\right)$ in the free area of the louver. The corresponding range of OA flow is 430 to $4960 \mathrm{cfm}\left(0.20\right.$ to $\left.2.34 \mathrm{~m}^{3} \mathrm{~s}^{-1}\right)$. The manufacturers data indicate that the pressure drop across the louver system (without a bird screen) ranges nonlinearly from $2.5 \mathrm{~Pa}$ with an air velocity through the free area of $470 \mathrm{fpm}\left(2.4 \mathrm{~m} \mathrm{~s}^{-1}\right)$ to $747 \mathrm{~Pa}$ with a velocity of $7300 \mathrm{fpm}\left(37 \mathrm{~m} \mathrm{~s}^{-1}\right)$. 


\section{Results}

Figure 4 shows the \%error of MT1 plotted versus the reference OA flow rate. The figure includes results of tests with a range of \%OA and with a range of OA damper positions. The measured pressure signal from the airflow sensor blades of MT1 are also plotted. With our research-grade pressure transducer used to measure this pressure difference, MT1 is accurate within approximately $\pm 20 \%$ for outside air flow rates exceeding approximately $400 \mathrm{cfm}\left(0.19 \mathrm{~m}^{3}\right.$ $\left.\mathrm{s}^{-1}\right)$. At the $400 \mathrm{cfm}$ flow rate, the corresponding average velocity in the $1.24 \mathrm{ft}^{2}\left(0.12 \mathrm{~m}^{2}\right)$ of free area of the louver is $320 \mathrm{ft} \mathrm{min}^{-1}\left(1.6 \mathrm{~m} \mathrm{~s}^{-1}\right)$. With lower reference OA flow rates, the \%error of MT1 becomes variable and often very high, presumably because the pressure signal is very low, e.g., $<1 \mathrm{~Pa}$.

In actual applications, the pressure transducer normally used in conjunction with MT1 will be less accurate (and also less expensive) than our research-grade pressure transducer. Therefore, for three OA flow rates, Figure 4 includes sets of error bars illustrating the expected range of accuracy of MT1 with errors in differential pressure measurement of $\pm 1 \mathrm{~Pa}$ and $\pm 3 \mathrm{~Pa}$, which are consider more typical of the errors that occur with the electronic pressure transducers commonly used in field settings.

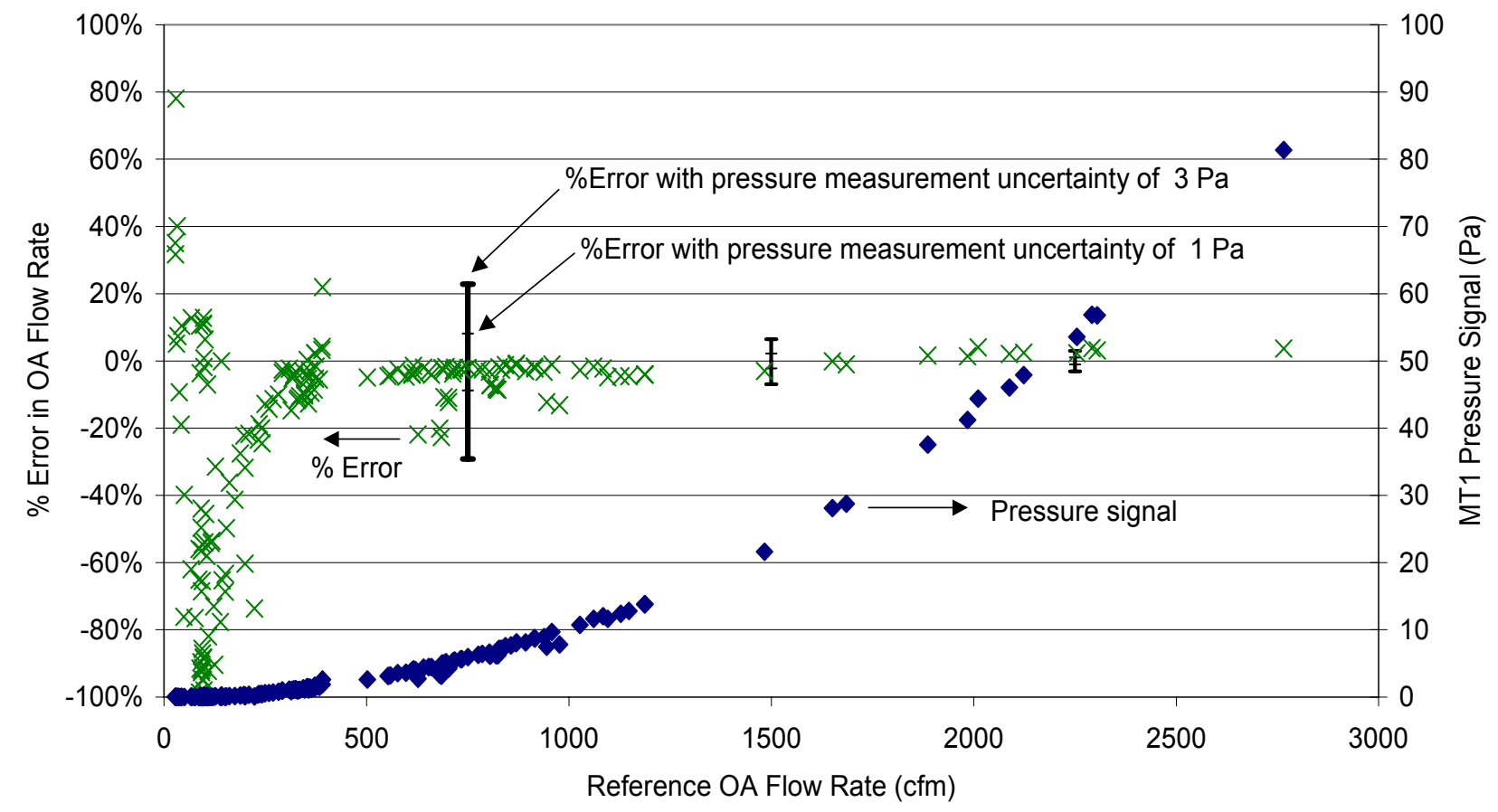

Figure 4. Accuracy and pressure drop of MT1 versus flow rate.

The accuracy of MT1, and the associated ranges in \% OA are illustrated in Figure 5. Figure 6 shows the accuracy versus OA air flow rate, with different data point markers displayed depending on the degree of opening ( $25 \%, 50 \%$ and $100 \%$ open) of the OA damper located just $0.5 \mathrm{ft}(0.15 \mathrm{~m})$ downstream of the downstream edge of the airflow sensor blades. From a visual inspection, the accuracy of MT1 appears to be nearly independent of both \% OA and OA damper position. 


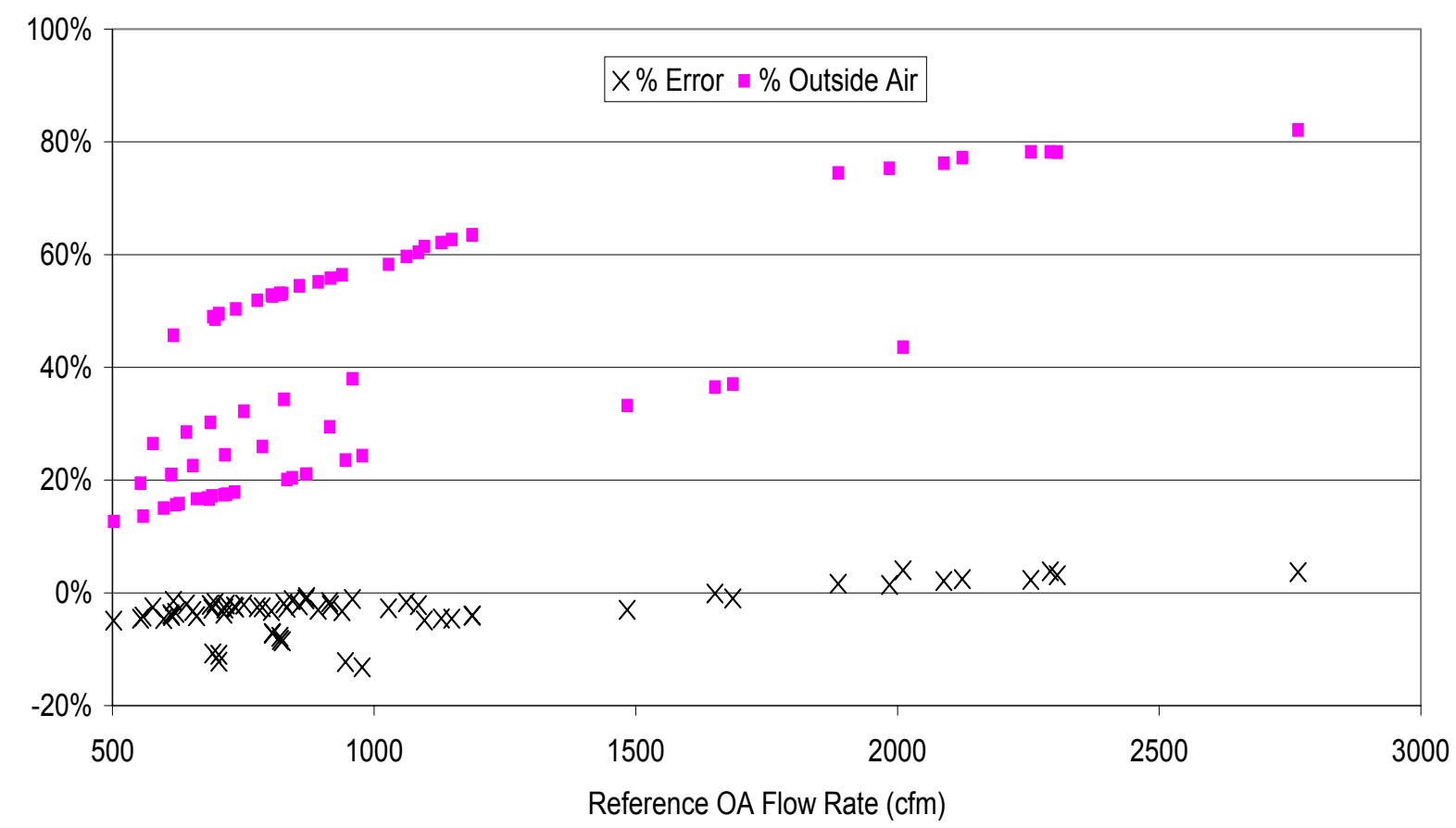

Figure 5. Accuracy of MT1 and \% OA during tests.

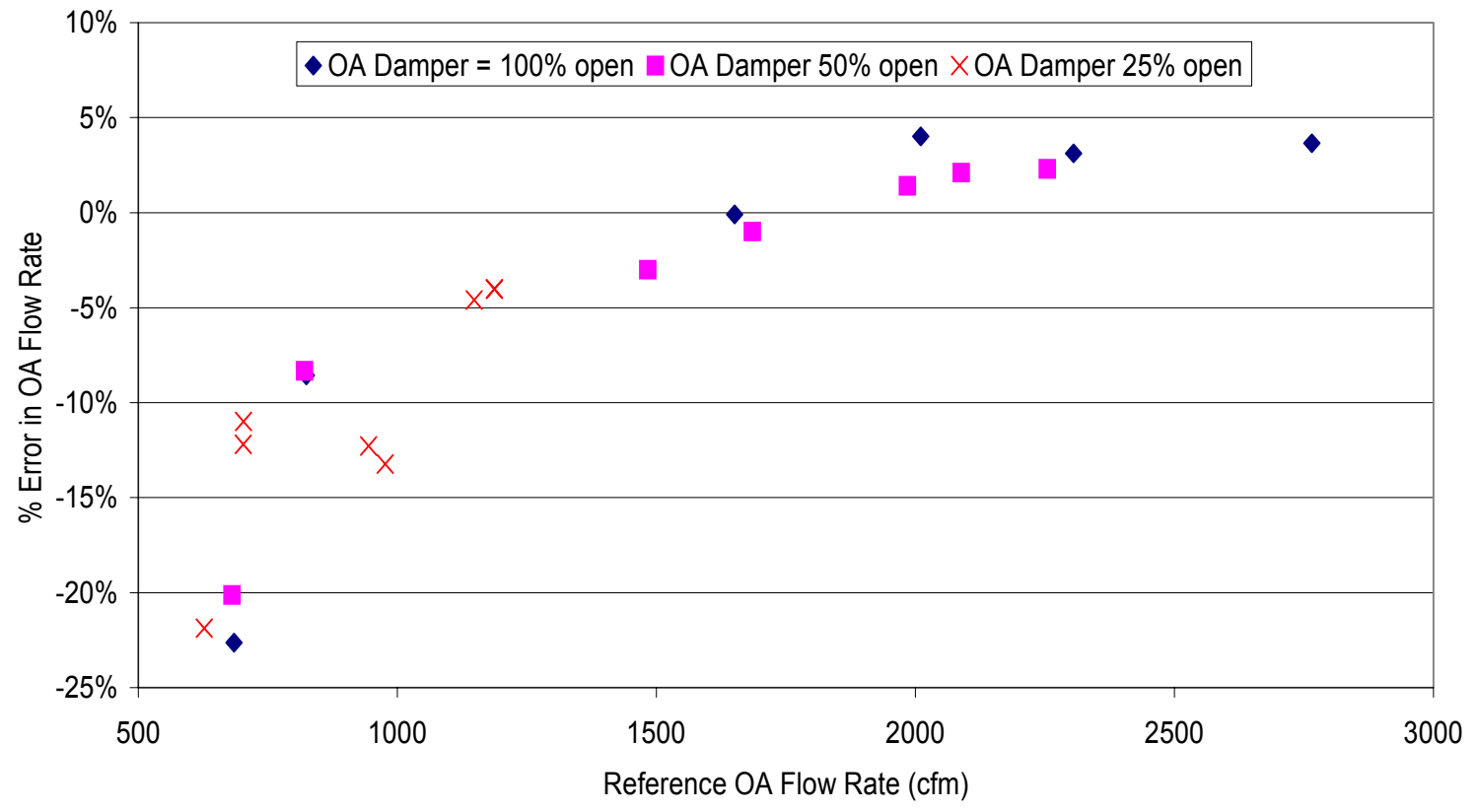

Figure 6. Accuracy of MT1 and OA damper position during tests.

Placement of wall surfaces near the outside air intake in the three configurations illustrated in Figure 2 had no discernable impact on the accuracy of MT1 (data not shown). The exception was 
a reduced accuracy in configuration 2 , emulating a visual screen, when dimension " a" was 0.33 $\mathrm{ft}(0.1 \mathrm{~m})$ or less. However, in practice visual screens will normally located be at least $3 \mathrm{ft}(1 \mathrm{~m})$ from the OA inlet to enable access.

The effects of directing 2 to $7 \mathrm{mph}\left(0.9\right.$ to $\left.3 \mathrm{~m} \mathrm{~s}^{-1}\right)$ simulated winds at the OA intake were variable. When the reference OA flow was $900 \mathrm{cfm}\left(0.42 \mathrm{~m}^{3} \mathrm{~s}^{-1}\right)$ with a corresponding average velocity of $720 \mathrm{fpm}\left(3.7 \mathrm{~m} \mathrm{~s}^{-1}\right)$ in the free area of the louver system, the simulated winds had a discernable but unimportant (few percent) impact on measurement accuracy. With a reference OA flow of $400 \mathrm{cfm}\left(0.19 \mathrm{~m}^{3} \mathrm{~s}^{-1}\right)$, which was our lower limit for $\pm 20 \%$ accurate measurements using a research grade pressure transducer, winds at a 45 degree angle increased measurement errors by up to 25 percentage points. Wind from the left side of the OA intake, when viewed from the top, caused the largest error. From inspection of the louver geometry depicted in Figure 3 , winds from right and left and right sides of the louver system would be expected to differentially impact velocities within the louver system.

The measured pressure drop across MT1 varied depending on the location of the downstream reference static pressure tap. We made independent measurements of pressure drop using static pressure taps on the two side walls and top wall of the OA inlet duct and the resulting pressure drops varied by approximately a factor of three. Figure 7 shows the average of the three measured pressure drops versus average velocity in the free area of the louver. For comparison, we also show a curve of pressure drop versus airflow based on the manufacturer's product literature. Our measured pressure drop data correspond well with those reported by the manufacturer; however, given the variability of our measurements with pressure tap location, the high level of correspondence may be fortuitous.

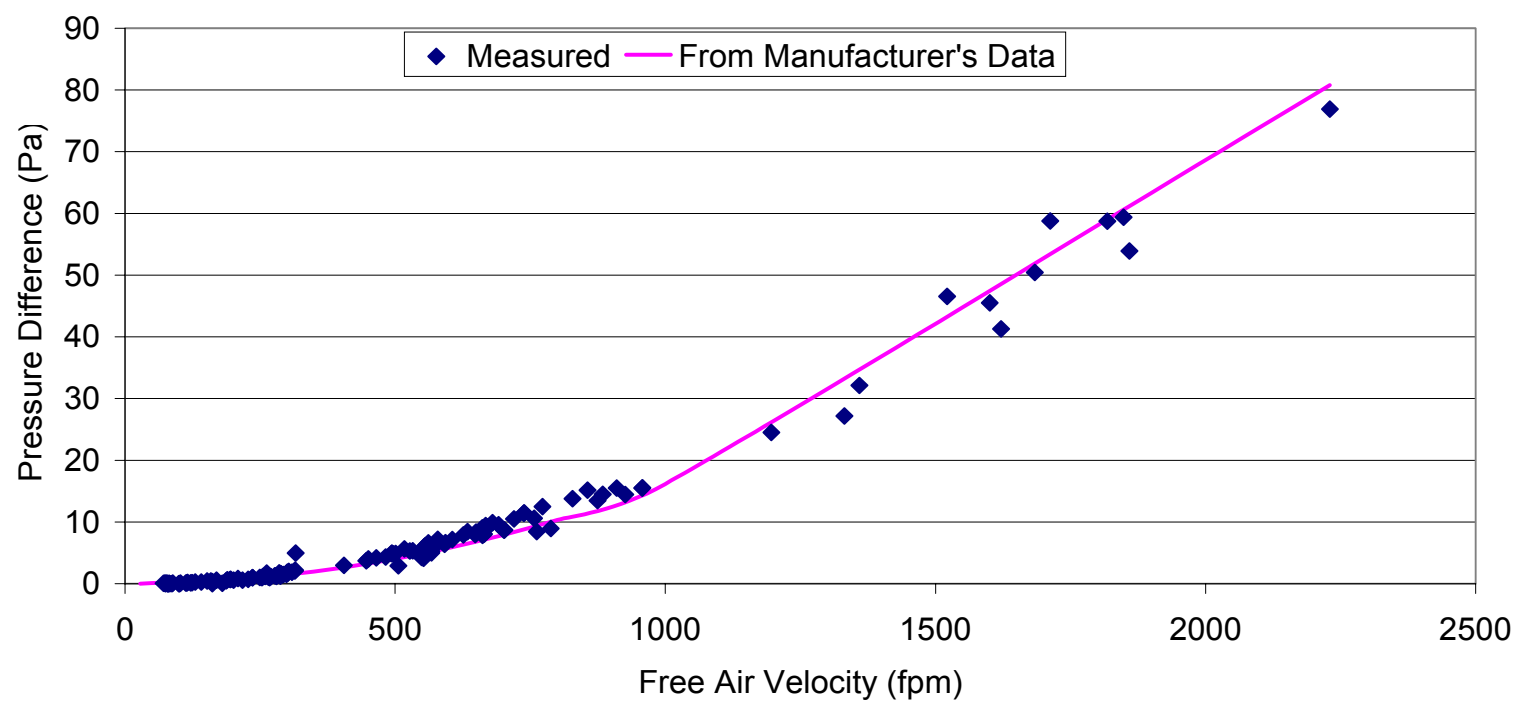

Figure 7. Pressure drop of air flowing through MT1.

\section{Discussion}

\section{Testing methods}

The test system and protocol developed for this project provided a convenient and accurate method of evaluating the accuracy of MT1 under conditions without wind, and should be equally suitable for many other OA flow measurement technologies. Individual data points could be 
obtained rapidly (e.g., within one minute) after flow rates were adjusted to obtain the desired conditions. If large numbers of tests were required, a computer control system could be developed to automatically adjust damper positions and fan speeds. Replacing the large reference flow meter with the smaller reference flow meter (or vice versa) requires approximately 15 minutes of labor. Removing and replacing the OA flow measurement technology in the experimental system may require up to several hours of labor, depending upon the technology. Further work is necessary to develop hardware and protocols for assessing the influence of winds on measurement system accuracy. In addition, to confirm the reliability of data obtained with simulated winds we believe that limited testing should be performed with the system exposed to real winds.

We are less confident of the hardware and procedure used to measure the static pressure drop across MT1, despite the correspondence of our data with manufacturers data. Flanges of different widths around the periphery of MT1 obstructed airflow; thus, we would expect the airflow to be separated from the duct walls near the static pressure taps. The flow separation and turbulent eddies downstream of the flanges would cause errors in measurements of pressure drop and may explain why the measured pressure drops varied by a factor of three depending on the selected downstream pressure tap. It may be preferable to measure static pressure drops in a special test system with a long straight duct containing no OA damper located downstream of the OA flow measurement technology.

Performance of Measurement Technology

The performance of MT1 under conditions without wind was encouraging. The accuracy of the OA flow rate measurement was approximately $\pm 20 \%$ or better for OA flow rates of 400 to 2700 $\operatorname{cfm}\left(0.19\right.$ to $\left.1.3 \mathrm{~m}^{3} \mathrm{~s}^{-1}\right)$. We have not yet surveyed potential users of OA measurement technologies to assess their accuracy requirements. However, considering the complete lack of a OA air flow rate measurement technology in most buildings and the imprecise knowledge of the relationship of OA ventilation rates with health, we anticipate that systems with an accuracy on the order of $\pm 20 \%$ will be considered valuable by users. The pressure drop of air flowing through MT1 was not atypical of the pressure drop in other louver systems that have no integral OA flow measurement system. Hence, the measurement of OA flow rate was accomplished with little increment in pressure drop, and the measurement system should not significantly increase fan energy consumption. The performance of MT1 under conditions with winds remains to be

determined. Also, the cost of MT1 could be a barrier to widespread adoption. We paid $\$ 800$ for the $2 \mathrm{ft}$ by $2 \mathrm{ft}$ louver system with integral airflow blades (without a pressure transducer).

\section{Conclusions}

The test system and protocol developed for this project provides a convenient and accurate method of evaluating the accuracy of technologies for measuring outside airflows into air handling systems. Further research is needed to develop systems and protocols for assessing the influence of winds on measurement accuracy.

This research has shown that MT1 can provide a reasonably accurate measurement of OA flow rate over a broad flow range, under conditions without wind. The measurement of OA flow rate was accomplished with minimal increase in airflow resistance.

\section{Acknowledgements}

This work was supported by the assistant Secretary for Energy Efficiency and Renewable Energy, Building Technologies Program of the U.S. Department of Energy under contract DE-AC03- 
76SF00098. The authors thank Mike Apte and Craig Wray for their reviews of a draft of this document.

\section{References}

ASHRAE (2001) ANSI/ASHRAE Standard 62-1999 - Ventilation for acceptable indoor air quality. American Society of Heating, Refrigerating, and Air Conditioning Engineers, Inc. Atlanta, GA

ASHRAE (1999a) ANSI/ASHRAE Standard 120-1999 (1999) Method of testing to determine

flow resistance of hvac ducts and fittings. ASHRAE, Atlanta, GA

ASHRAE (1999b)ANSI/ASHRAE 51-1999 (1999) Laboratory methods of testing fans for aerodynamic performance rating. ASHRAE, Atlanta, GA

Emmerich SJ and Persily AK (1998) Energy impacts of infiltration and ventilation in U.S. office buildings using miultizone airflow simultion. Proceedings of IAQ and Energy 98, pp. 191206, ASHRAE, Atlanta, GA.

Howell, RH and Sauer HJ (1987) Velocity variation effects on a rotating vane anemometer at coil faces. ASHRAE Transactions 93(2): 200-210

Kettler JP (1995) Minimum ventilation control for VAV systems: fan tracking versus workable solutions. ASHRAE Transactions 101(2): 625-630

Krarti, M, Brandemuehi MJ, Schroeder C, Jeannette E (1999) Techniques for measuring and controlling outside air intake rates in variable air volume systems. Final Report of ASHRAE Research Project RP-980, ASHRAE, Atlanta.

Lagus Applied Technologies (1995) Air change rates in non-residential buildings in California, Report P400-91-034BCN, California Energy Commission, Sacramento, CA.

Orme, M. (1998) Energy impact of ventilation, Technical Note 49, International Energy Agency - Air Infiltration and Ventilation Centre, Coventry, Great Britain.

Persily A (1989) Ventilation rates in office buildings. Proceedings of the IAQ'89 Conference The Human Equation: Health and Comfort, pp. 128-136., ASHRAE, Atlanta.

Turk, B.H., Brown J.T., Geisling-Sobatka, K., Froelich, D.A., Grimsrud, D.T., Harrison, J., Koonce, J.F., Prill, R.J., and Revzan, K.L. (1987) Indoor Air Quality and Ventilation Measurements in 38 Pacific Northwest Commercial Buildings--Volume 1: Measurement Results and Interpretation, Lawrence Berkeley Laboratory Report, LBL-22315 1/2, Berkeley, CA. 